\title{
Pompe à chaleur sur nappe et héliogéothermie Une opération pilote sur 224 logements à Aulnay-sous-Bois
}

\section{Heat pump on ground water and combined solar/geothermal power A pilot operation on 224 dwellings at Aulnay-sous-Bois}

\author{
P. Iris
}

Centre d'informatique géologique

Ecole nationale supérieure des mines de Paris

\footnotetext{
Le dispositif présenté, qui à fait l'objet d'un brevet, est mis en auvre à Aulnay-sous-Bois, sur un ensemble de 224 logements collectifs neufs.

La nappe réservoir sableuse, d'âge Eocène inférieur, est située entre 60 et $80 \mathrm{~m}$ de profondeur. à la température de $13^{\circ} \mathrm{C}$. Il s'agit d'un horizon tout à fait adapté aux im. meubles collectifs, qui couvre l'ensemble nord de la région parisienne.

Cette installation est exploitée depuis un an et un premier bilan complet en est présenté.
}

The system described, which is patented, is in use at Aulnay-sous-Bois on a group of 224 new flats.

The sandy water basin of the lower eocene epoch is located at a depth between 60 and $80 \mathrm{~m}$ and is at $a$ temperature of $13^{\circ} \mathrm{C}$. This horizon is perfectly suited to apartment buildings which cover the entire north part of the Paris area.

This facility has been in operation for a year and the first complete record is given.
Le stockage thermique en nappe souterraine est l'objet de travaux de Recherche depuis plusieurs années sous l'impulsion plus particulière du Plan Construction et de l'ancienne Délégation générale à la Recherche scientifique et technique.

Le Centre d'informatique géologique de l'École des mines de Paris s'est spécialisé dans la modélisation mathématique des transferts dans le sous-sol et en particulier des transferts thermiques. C'est à ce titre qu'il a organisé en liaison avec EDF et avec le soutien financier du Plan Construction une première expérience de stockage thermique d'envergure en 1977 dans une nappe peu profonde, dans le but essentiel de contrôler sur le terrain la validité des méthodes de calcul (expérience de Campuget, dans le Gard).

Suite à ces travaux, et en réponse à un nouvel appel d'offre lancé en 1980 par le Plan Construction et l'ancien
Commissariat à l'énergie solaire sur le thème du stockage saisonnier de calories solaires dans la perspective d'opérations de démonstration, il a été proposé de mettre en œuvre le dispositif du "doublet héliogéothermique " dans le cadre d'une opération immobilière réelle portant sur 224 logements collectifs neufs à Aulnay-sous-Bois en SeineSaint-Denis. Ce système qui a pour objectif de permettre un large développement de l'utilisation par pompe à chaleur d'une nappe régionale peu profonde de la région parisienne, a été proposé dans l'esprit de valoriser ces travaux de recherche dans le cadre de techniques de chauffage susceptibles de se développer dans un avenir proche. L'opération a été rendue possible grâce au concours du Maître d'ouvrage, le Foyer du fonctionnaire et de la famille, et au soutien financier du Plan Construction, de l'Agence française pour la maîtrise de l'énergie, et de la Commission des communautés européennes. 


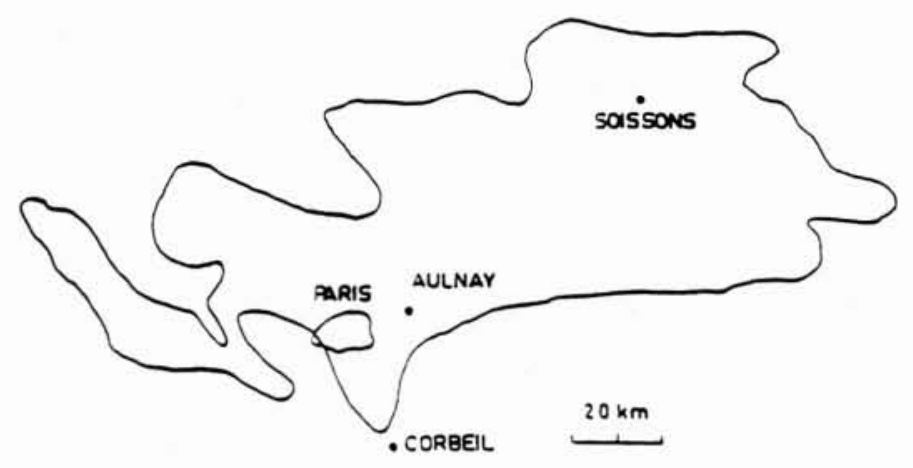

Figure 1. - Extension générale de la nappe de I'Yprésien.
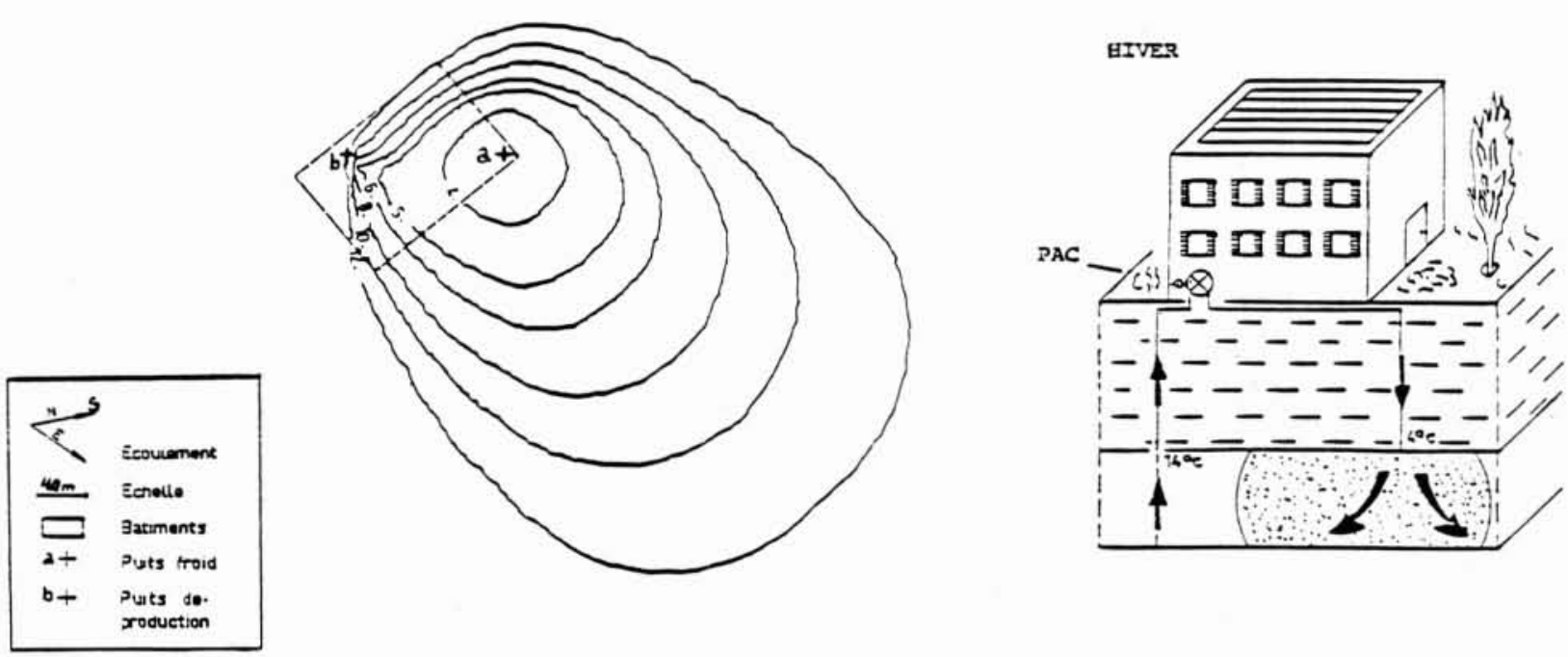

Figure 2. - Isothermes dans la nappe après 20 ans d'exploitation.

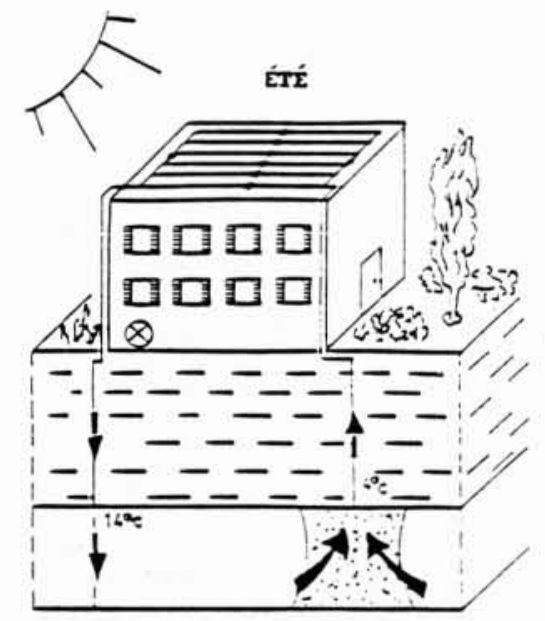

Figure 3. - Schéma de principe du doublet héliogéothermique.

Figure 4. - Isothermes dans la nappe après le $20^{\circ}$ cycle annuel. 
Les nappes souterraines : une source de chaleur pour les pompes à chaleur

Les nappes souterraines sont constituées d'une matrice poreuse saturée dont la structure permet la circulation et le pompage de l'eau. L'eau et la matrice sont à l'équilibre thermique à une température dans la plupart des cas constante, qui résulte d'un emmagasinement naturel de chaleur sous les actions conjuguées des échanges thermiques à la surface du sol, de l'infiltration des eaux de pluie et du flux géothermique. Les nappes superficielles sont à la température moyenne annuelle de l'air (de 10 à $15^{\circ} \mathrm{C}$ selon la région), l'augmentation avec la profondeur étant de $3{ }^{\circ} \mathrm{C}$ par $100 \mathrm{~m}$ (gradient géothermique moyen). Elles sont largement réparties sur le territoire national et constituent une source de chaleur à très basse température tout à fait adaptée à l'utilisation des pompes à chaleur dans le cadre du chauffage domestique et du tertiaire, le logement collectif moyen ou pavillonnaire regroupé représentant le "marché " principal pour l'habitat.

\section{Un exemple en région parisienne : la nappe de l'Yprésien (figure 1)}

En Région Parisienne, la nappe dite de l'Yprésien en est un exemple : il s'agit d'un horizon aquifère sablo-argileux limité à sa base par des argiles et à son toit par des calcaires le mettant en pression, qui couvre le Nord de la Région Parisienne : la nappe est située en général entre $80 \mathrm{~m}$ et $100 \mathrm{~m}$ de profondeur, sa température est de $13^{\circ} \mathrm{C}$ et le débit par forage est en moyenne de 60 à $80 \mathrm{~m}^{3} / \mathrm{h}$. On peut y prélever une source froide de pompe à chaleur de l'ordre de $600 \mathrm{~kW}$ et raccorder environ 300 logements collectifs par forage. Bien reconnue en région parisienne, la nappe est particulièrement productive dans le Nord de Paris, en Seine-Saint-Denis, dans le Val d'Oise, au Nord de la Seine-et-Marne et au Nord-Est des Yvelines.

\section{Quelle méthode d'exploitation?}

La question qui se pose est de savoir comment exploiter cette ressource dans une perspective de large développement.

La première solution est de pomper l'eau, en extraire les calories par la pompe à chaleur et la rejeter dans un réseau de surface.

Cette solution, si elle a le mérite de la simplicité n'est pas toujours techniquement possible à mettre en œuvre, en particulier dans le cas où le réseau d'assainissement n'est pas séparé du réseau pluvial, et présente l'inconvénient d'exploiter une ressource souterraine dont le taux de renouvellement naturel est faible en regard des besoins potentiels. Si elle peut être mise en œuvre ponctuellement, un large développement de cette méthode conduirait inévitablement à des désordres et à des conflits avec les usages traditionnels de l'eau souterraine.

La seconde solution est de réinjecter dans la nappe, par l'intermédiaire d'un second forage convenablement situé par rapport au premier, l'eau refroidie par la pompe à chaleur. De cette façon, on se contente de prélever la chaleur contenue dans l'eau, sans altérer le bilan hydraulique général de la ressource souterraine.

Sur le plan technique, il faut s'assurer qu'il n'y a pas de possibilité d'interférence thermique entre puits de réinjection et puits de production, avec à terme, le risque d'une chute de la température au puits de production.

Dans ce domaine, les modèles de simulation des transferts thermiques en nappe permettent d'étudier la faisabilité d'un projet. Ces calculs ont été effectués dans le cadre de l'opération immobilière d'Aulnay-sous-Bois de 224 logements collectifs.

La figure 2 montre la répartition (théorique et calculée par modèle mathématique) de la température dans la nappe après 20 ans d'exploitation par cette méthode, avec un puits de production (b) et un puits de réinjection (a). Les traits pointillés symbolisent l'emprise au sol des bâtiments. On s'aperçoit que compte tenu de la configuration locale, la perturbation dans la nappe va bien au-delà de l'emprise au sol des logements, et qu'une chute de température se produit au puits de production (b). La méthode de réinjection pose donc un problème de protection thermique du milieu souterrain, en particulier en zone urbaine où les distances d'implantation des puits risquent d'être faibles et les terrains limités en regard des volumes et des énergies mises en œuvre.

\section{Le doublet héliogéothermique}

Le système du doublet héliogéothermique est proposé pour pallier ces inconvénients dans les nappes à faible vitesse naturelle d'écoulement, comme c'est en général le cas pour la nappe de l'Yprésien.

L'hiver, l'eau est pompée au puits de production (b) et réinjectée au puits (a) après extraction de ses calories par la pompe à chaleur. L'été, le régime hydraulique du doublet est inversé. L'eau froide est répompée puis réinjectée au puits de production après avoir été réchauffée par l'intermédiaire de batteries d'échange sur l'air fonctionnant en convection naturelle : l'eau est portée de $5^{\circ} \mathrm{C}$ à $14^{\circ} \mathrm{C}$ grâce à l'air, qui l'été a une température moyenne de $18^{\circ} \mathrm{C}$ en région parisienne. Ce procédé permet d'éviter un développement trop important du front froid dans la nappe et assure une température constante au puits de production, comme le montre la figure 4, résultat d'une simulation mathématique du "doublet héliogéothermique " appliqué au cas précédent.

Dans l'habitat, ce système nécessite l'installation supplémentaire d'échangeurs sur l'air ou de capteurs solaires simplifiés qui permettent d'assurer ainsi une sorte de "garantie et de perennité thermique de la ressource" pour l'utilisateur lui-même et pour son voisinage. Il peut s'implanter facilement dans le cadre du tertiaire par exemple où, aux besoins de chauffage l'hiver, s'ajoutent des besoins de climatisation l'été. 


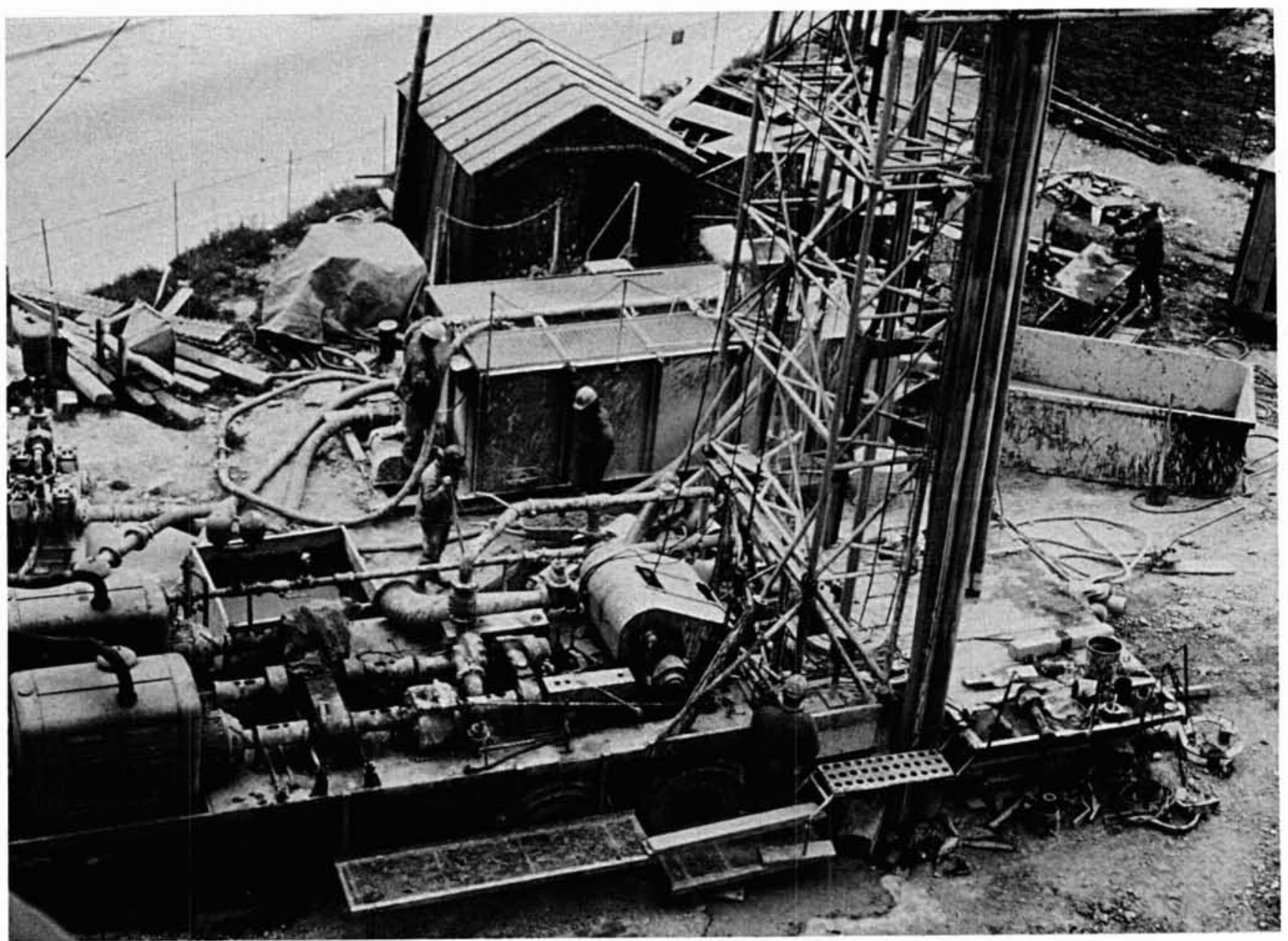

Figure 5. - Le chantier de forage.

\section{La réalisation expérimentale d'Aulnay-sous-Bois}

Ce système a été mis en œuvre dans le cas de l'opération immobilière citée précédemment avec deux objectifs :

- un objectif à moyen terme qui consiste à mettre en œuvre concrètement le concept du doublet héliogéothermique, à contrôler le comportement des batteries d'échange sur l'air, l'impact thermique sur la nappe, etc.;

- un objectif à plus court terme qui consiste simplement à étudier sur un cas réel le comportement de la nappe en régime de pompage et de réinjection, à optimiser le système et ses régulations, et à tirer des bilans sur les plans technique, énergétique et économique d'une opération de pompe à chaleur en habitat collectif.

L'installation comporte deux forages de $87 \mathrm{~m}$ de profondeur, distants de $120 \mathrm{~m}$ et équipés chacun d'une pompe immergée débitant $50 \mathrm{~m}^{3} / \mathrm{h}$ et d'un dispositif de réinjection par tube plongeur (le température de l'eau de la nappe est localement de $12,7^{\circ} \mathrm{C}$ ).

Le chauffage est assuré par deux pompes à chaleur fournissant $500 \mathrm{~kW}$ thermiques pour une puissance nominale de déperdition des bâtiments de $900 \mathrm{~kW}$. Un appoint au fuel est prévu, la chaleur est distribuée par planchers chauffants à basse température et radiateurs thermostatés (température de départ $45^{\circ} \mathrm{C}$ pour une température extérieure de $-7{ }^{\circ} \mathrm{C}$ ).

L'eau chaude sanitaire est fournie en totalité à $50^{\circ} \mathrm{C}$ par une pompe à chaleur particulière de $160 \mathrm{~kW}$ thermique et un système de stockage de $20 \mathrm{~m}^{3}$ permettant d'exploiter les " heures creuses" de la tarification EDF.

La recharge saisonnière de la nappe est assurée par des batteries d'échange statique sur l'air extérieur fonctionnant en convection naturelle et constituées d'une superposition d'éléments d'échange à trame de cuivre (capteurs climatiques à fort coefficient d'échange sur l'air) à raison de $6 \mathrm{~m}^{2}$ de composants par logement. La régulation prévoit également la possibilité d'utiliser, en période de chauffe, ces capteurs, en complément de la nappe à la source froide des pompes à chaleur.

Le bilan d'investissement de cette opération de 224 logements est le suivant (valeur au logement 1981 H.T.) :

- Distribution chauffage et eau chaude sanitaire

: $7800 \mathrm{~F}$.

- Forages + échangeurs + pompes immergées

- Pompe à chaleur, boucle froide, régulation, équipement, centrale de chauffe

$6100 \mathrm{~F}$.

- Echangeurs atmosphériques 


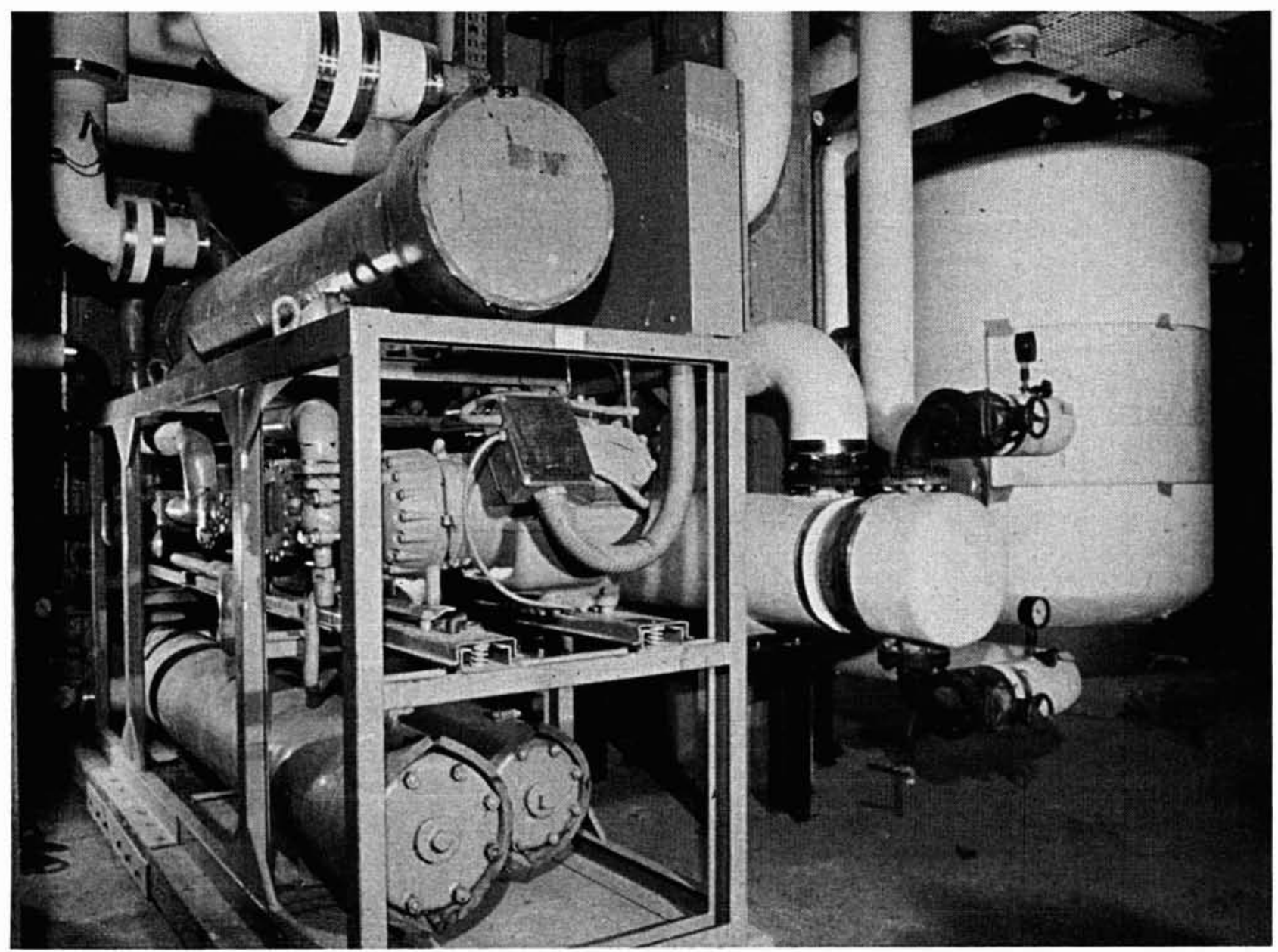

Figure 6. - Une pompe à chaleur.

- Surcoût génie civil (centrale de chauffe, terrasse, accès forages)

- Coût total

- Surcoût par rapport à une installation classique avec radiateurs et chauffage au fuel domestique

: $2200 \mathrm{~F}$

: $22600 \mathrm{~F}$

$: 15800 \mathrm{~F}$

Avec le bilan prévisionnel d'exploitation au logement :

Besoins annuels chauffage

et eau chaude sanitaire $=9700 \mathrm{kWh}$

tep économisée $=0,8$ tep

Tonne de fuel déplacée $=1,4 \mathrm{t}$.

Total électricité consommée pour le chauffage et l'eau chaude sanitaire par le système $=2800 \mathrm{kWh}$

COP global $=3,4$

Economie annuelle d'exploitation par rapport au fuel domestique (y compris frais de maintenance supplémentaires) $=1900 \mathrm{~F}$. TTC soit $50 \%($ val. 81$)$

Temps de retour brut du surinvestissement $=10$ ans

\section{Bilan actuel}

L'installation a été mise en service le 30 mars 1983, à la fin de la campagne de chauffe 1982-1983; les premiers essais de fonctionnement ont été mis à profit pour effectuer les réglages alors que les logements étaient encore pratiquement inoccupés.

La première campagne de chauffe complète a débuté le 14 octobre 1983, et a été l'objet d'une campagne de mesure détaillée. Les premiers bilans, pour la période du 18 octobre 1983 au 24 avril 1984 sont les suivants :

Température extérieure moyenne de la période $=6^{\circ} \mathrm{C}$ Energie fournie pour le chauffage $=1787 \mathrm{MWh}$ Appoint fuel $=202 \mathrm{MWh}$

Energie électrique absorbée par les pompes à chaleur chauffage $=366 \mathrm{MWh}$

Coefficient de performance brut $=4,3$

Energie fournie pour l'eau chaude sanitaire (distribution moyenne à $48{ }^{\circ} \mathrm{C}$, volume d'eau chaude $=4374 \mathrm{~m}^{3}$, pertes thermiques de la boucle de distribution $=138 \mathrm{MWh})=$ $323 \mathrm{MWh}$

Energie électrique absorbée par la pompe à chaleur ECS $=111 \mathrm{MWh}$

COP brut $=2,73$ 
Consommation des auxiliaires $=76 \mathrm{MWh}$

Coefficient de performance global pour la période $=3,41$

Taux de couverture par les PAC $=90 \%$

Tonnes de fuel déplacés (rendement chaufferie $=70 \%$ )

$=235$ tonnes

Economie par logement $=2060 \mathrm{~F}$ TTC, soit $50,5 \%$ d'exploitation (en tenant compte des surcoûts de maintenance) par rapport au fuel lourd.

Les graphes ci-contre représentent pour la période correspondante à laquelle sont ajoutés quelques jours supplémentaires de chauffage en mai 1984 , les bìlans mois par mois pour le chauffage et l'eau chaude sanitaire (pour cette dernière, on prend également en compte l'énergie nécessaire au maintien en température de la boucle de distribution).

On distingue les auxiliaires froids, c'est-à-dire les pompes immergés et les pompes nécessaires au fonctionnement des pompes à chaleur, et les auxiliaires chauds qui représentent les pompes de distribution, qui dans l'ensemble auraient également été mis en œuvre dans le cadre d'un chauffage centralisé classique.

D'une façon générale, les résultats de cette première campagne de chauffe sont voisins de ce qui était attendu.

La part d'appoint pour le chauffage est plus forte que prévu du fait de la régulation adoptée qui a consisté, pendant les heures de pointes d'hiver ( 4 heures par jour) à substituer aux pompes à chaleur l'appoint fuel. En fait, il a été mis en évidence que l'inertie des bâtiments permettaient de limiter le recours à cet appoint.

La forte consommation de fuel pour la production d'eau chaude sanitaire en fin de saison est due à la panne d'un des compresseurs de la PAC ECS à cette période.

Le principal problème est venu de la difficulté de réguler en fonction des besoins, le débit de la pompe immergée, à l'aide d'une chaîne de régulation comprenant en particulier un variateur électronique commandant la vitesse de rotation du moteur de la pompe immergée.

Cette difficulté technique est en voie de résolution, mais a entraîné d'une part, une surconsommation (la pompe immergée ayant fonctionné en quasi permanence à son débit maximal) et d'autre part, a limité, du fait du processus de régulation de l'installation, la part relative d'énergie de source froide fournie par les capteurs : il était prévu que la nappe fournisse $55 \%$ de cette énergie, les capteurs $45 \%$; or cette saison, le rapport a été de $90,5 \%$ pour la nappe et $9,5 \%$ pour les capteurs.

La nappe a, par conséquent, été plus sollicitée en hiver que prévu et, de ce fait, la recharche saisonnière a été partielle, compte-tenu du dimensionnement des capteurs : du 14 octobre 1983 au 4 juin 1984, environ $210000 \mathrm{~m}^{3}$ ont été prélevés à la nappe puis réinjectés à $7,2^{\circ} \mathrm{C}$ en moyenne. La recharge thermique a débuté le 06 juillet et, jusqu'au 28 septembre, $80000 \mathrm{~m}^{3}$ ont été repompés et réinjectés au puits de production à $12,7{ }^{\circ} \mathrm{C}$ en moyenne.

Sur le plan hydrodynamique, aucun colmatage des forages n'a été observé. Le débit spécifique au puits de réinjection d'hiver a été maintenu aux environs de $8 \mathrm{~m}^{3} / \mathrm{h} / \mathrm{m}$ (débit injecté correspondant à une remontée d'eau de $1 \mathrm{~m}$ au forage qui permet de mesurer les pertes de charge dans la nappe en injection) grâce à la mise en œuvre hebdomadaire de repompage pendant 1 heure au débit maximal (décrassage). En fin de saison, nous avons arrêtés les décrassages hebdomadaires, le débit spécifique
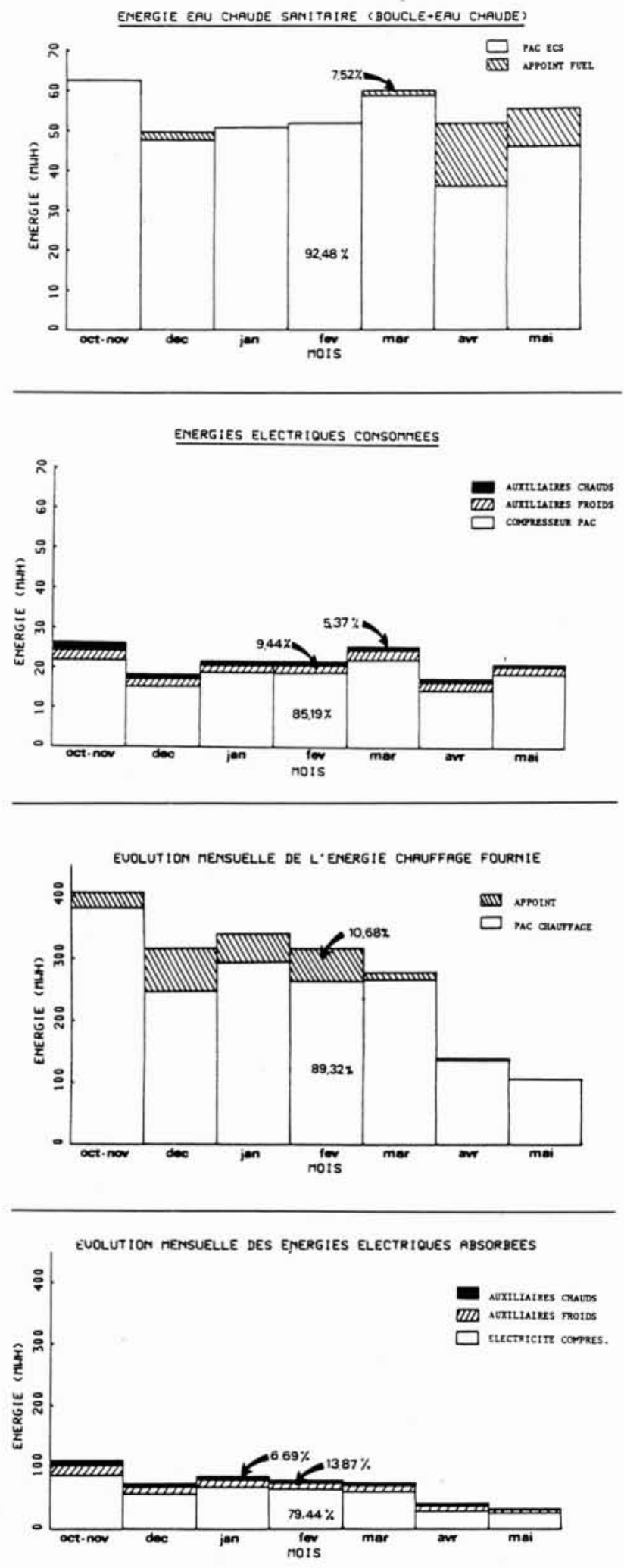

s'est stabilisé à $7 \mathrm{~m}^{3} / \mathrm{h} / \mathrm{m}$, pour une valeur initiale de $9 \mathrm{~m}^{3} / \mathrm{h} / \mathrm{m}$ à la livraison de l'ouvrage. Au puits de réinjection été, aucun pompage de décolmatage n'a été mise en œuvre et le débit spécifique de réinjection s'est maintenu à sa valeur initiale de $14 \mathrm{~m}^{3} / \mathrm{h} / \mathrm{m}$ 


\section{Conclusion}

Le suivi de l'installation pendant sa première année de fonctionnement a confirmé les bilans économiques prévisionnels.

Le temps de retour brut de l'investissement est dans notre cas compris entre 9 et 10 ans pour une opération qui n'a pas été dimensionnée de façon optimale sur ce plan, compte-tenu en particulier du nombre relativement faible de logements raccordés. L'analyse des postes d'investissement fait apparaître que le poids des investissements liés au système de distribution de chauffage, aux pompes à chaleur et aux forages correspond à un temps de retour de 7 ans, les capteurs permettant la recharge thermique de la nappe ajoutant 3 ans.

D'une façon générale, l'intérêt économique de ce type de dispositif dépend du coût d'accès à la nappe (qui dépend lui-même de la profondeur et de la productivité de la nappe) et du nombre de logements raccordables par doublet de forage.

Dans le nord de l'agglomération parisienne, pour des opérations de 500 logements, on peut, sur la nappe de l'Yprésien, envisager des opérations dont le temps de retour ne dépasse pas 5 ans pour une opération classique avec un doublet sans recharge, et 7 à 8 ans si l'on y ajoute les capteurs; dans ces conditions, la nappe de l'Yprésien représente une cible énergétique régionale économiquement viable qui pourrait être avantageusement exploitée en complément de la géothermie à grande profondeur, et qu'un tel dispositif héliogéothermique permettrait de préserver.

Sur le plan technique, les pompes à chaleur ont fonctionné comme prévu, le pompage et la réinjection n'ont pas posé de problèmes particuliers; certaines régulations, imparfaites, n'ont toutefois pas permis d'assurer la recharge thermique saisonnière, telle qu'elle avait été envisagée initialement. Leur réglage définitif est en cours et il est proposé à l'A.F.M.E. d'assurer le suivi de l'opération pour la seconde année de fonctionnement en 1985; l'installation fonctionnant avec l'ensemble de ses régulations. On mettra l'accent sur l'étude du comportement hydrodynamique et thermique de la nappe, avec le suivi en particulier des propriétés physico-chimique et bactériologique de l'eau de la nappe.

\section{Références :}

\section{Rapport LHM/RD/82/2}

Rapport CIG-Ecole des Mines pour le compte de CCE - Plan Construction - A.F.M.E. : "Définition d'un dispositif de chauffage par PAC et héliogéothermie ". 
L'opération d'Aulnay a été fortement influencée par deux contraintes extérieures : l'architecture qui avait été définie indépendamment et l'emprise foncière qui limitait la surface disponible. Ainsi la distance entre les deux puits est relativement faible (question de M. BLUMENTAL). Ce qui a permis de mettre en évidence la nécessité d'une recharge saisonnière pour assurer la pérennité de la ressource (M. MARIE). La recharge s'effectue à basse température.

De nombreux systèmes pouvaient être envisagés pour recharger la nappe cependant, le seul espace disponible étant en terrasse, l'étude comparative fut limitée à différents types de capteurs; type piscine, type radiateur fini-métal,... Si les aspects énergétiques, architecturaux et d'urbanisme avaient été pris en compte dès le départ de l'opération, d'autres solutions auraient pu être étudiées : capteurs intégrés aux bâtiments, captage par un étang décoratif (proposition de M. GRJEBINE). Le système mis en place a été retenu car il est peu coûteux (équivalent aux capteurs de type piscine) et permet de densifier la prise d'énergie au mètre carré d'emprise au sol. L'utilisation d'un étang décoratif est une sophistication qu'un maitre d'ouvrage normal risque de ne pas pouvoir se payer, à moins qu'on puisse envisager des opérations où il y ait des subventions qui permettraient de payer les capteurs (M. IRIS).

Ce surcoût ne profite qu'à la préservation de la ressource (question de $M$. GRENET) et n'a qu'un effet très marginal sur le rendement de l'opération, au niveau du COP des pompes à chaleur. COP : terme anglo-saxon usuel correspondant au CDP - Coefficient de performance en français.

Vouloir préserver l'équilibre thermique de la nappe est une ambition limitée mais " cela permettra à l'avenir d'envisager une densification maximale de l'exploitation des PAC sur nappe captive, avec garantie de la ressource. Et dans le cas où une collectivité locale s'assurerait de la maitrise de l'exploitation du gisement de chaleur dans son sous-sol, il lui serait en sus possible d'assurer cette exploitation en connectant plusieurs doublets entre eux, en réseau, d'où une plus grande souplesse de gestion, la possibilité de réduire les puissances de secours et une fiabilité accrue au meilleur coût " ( $M$. MARIE $)$.
Cet aspect " concept de recherche futuriste " de l'opération a été engendré par les contraintes foncières; s'il y avait eu $50 \mathrm{~m}$ de plus entre les puits, il n'y aurait pas eu de problèmes d'interférence d'un puits sur l'autre et l'on aurait généré une perturbation à l'aval. Son ampleur aurait été limitée aux environs immédiats du doublet (au bout de 20 ans, la température aurait baissée de $10{ }^{\circ} \mathrm{C}$ à 600 ou $700 \mathrm{~m}$ ) et n'aurait pas eu d'incidence sur la station de pompage d'eau d'Aulany située à $2,5 \mathrm{~km}$ (question de $M$. de VULPILLIERES). Les problèmes d'interférence ne sont importants qu'en cas de forte densité des installations; des précautions sont alors à prendre autour des stations de pompage. Pour les définir, on dispose d'outils de calcul, des modèles mathématiques de simulation dans lesquels on peut avoir une certaine confiance car depuis 1975 ils ont été recalés par rapport à l'expérience.

Ces mêmes modèles seront utilisés dans l'opération d'Aulnay pour estimer l'évolution de la nappe dans le temps (question de M. DESSUS - Président. Celle-ci est assez bien connue (épaisseur, vitesse et direction de l'écoulement,...) car des mesures ont été faites dans les puits du doublet. Le principal paramètre manquant est le coefficient de dispersion qui traduit le degré d'hétérogénéité de la nappe. Il sera estimé en calant les résulats obtenus grâce aux modèles sur la courbe de repompage des eaux froides en été. Des mesures pourront être faites dans les puits en période d'arrêt pour estimer d'éventuels changements de tous les paramètres.

Pour terminer il faut noter "qu'il existe une procédure AQUAPAC, qui est peut-être insuffisante à certain point de vue, mais, il serait intéressant, pour de grandes opérations suffisamment significatives, de lancer l'idée de garantir la pérennité de la ressource d'une part, par un suivi par des exploitants, des ingénieurs-conseil en exploitation du sous-sol des ouvrages, et également de proposer une étude d'impact à l'aide des moyens dont disposent les Pouvoirs Publics.

On peut, avec les outils de calcul dont on dispose maintenant, se faire une idée de l'impact des réinjections dans la nappe. Il serait peut être bon de lier la procédure AQUAPAC à des études d'impact sur le plan thermique pour chercher à éviter les interférences. Il serait dommage de ne pas utiliser cette possibilité pour organiser la gestion des nappes ».

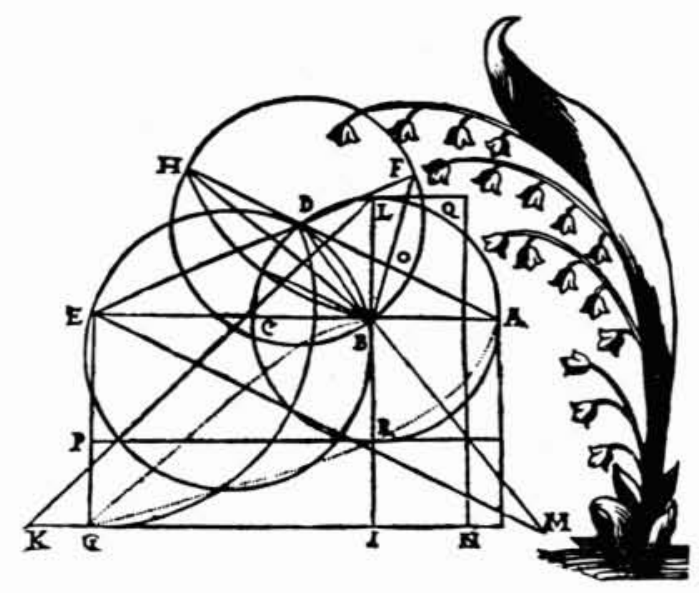

